ing status, and cancer history, as well as history of heart failure, myocardial infarction, or stroke. We believe that by including these characteristics in our model we have accounted for frailty with regard to mortality, and therefore we have not performed the additional requested sensitivity analyses.

Rhonda M. Cooper-DeHoff, PharmD, MS

dehoff@cop.ufl.edu

Yan Gong, PhD

Carl J. Pepine, MD

College of Pharmacy

University of Florida

Gainesville

Financial Disclosures: Dr Cooper-DeHoff reported receiving research funding from Abbott Laboratories during the conduct of INVEST and currently receiving funding from the National Institutes of Health. Dr Pepine reported receiving research grants from the National Heart, Lung, and Blood Institute (NHLBI), Abbott Laboratories, Baxter, Pfizer, GlaxoSmithKline, and Bioheart Inc; serving as consultan for Abbott Laboratories, Forest Laboratories, Novartis/Cleveland Clinic, NicOx, An globlast, Sanofi-Aventis, NHLBI, National Institutes of Health, Medtelligence, and SLACK Inc; and receiving unrestricted educational grants from AstraZeneca AtCor Medical Inc, Daiichi Sankyo Inc, Eli Lilly, Pfizer Inc, Sanofi-Aventis, and Schering-Plough. No other disclosures were reported.

1. Denardo SJ, Gong Y, Nichols WW, et al. Blood pressure and outcomes in very old hypertensive coronary artery disease patients: an INVEST substudy. Am J Med. 2010;123(8):719-726.

\section{Effect of Oral Glucosamine on Pain-Related Disability in Patients With Chronic Low Back Pain}

To the Editor: In their randomized placebo-controlled trial, Mr Wilkens and colleagues ${ }^{1}$ studied the effect of oral glucosamine on pain-related disability in patients with chronic low back pain (LBP). The authors concluded that, in a representative sample of the LBP population presenting in general clinical practice, oral glucosamine did not provide any advantages over placebo for self-administered functional, pain-related, and quality-of-life outcome measures.

However, based on the information given in the article, we find it hard to assess the generalizability of the study findings. The authors described that recruitment occurred via general practitioners, physiotherapists, chiropractors, and self-referrals through a newspaper advertisement. It would be helpful to know the distribution of included participants among these 4 methods of recruitment. Self-referred participants had to meet the additional requirement of a recent magnetic resonance imaging (MRI) scan, but it would be helpful to have more information about the Norwegian health care system to understand the implications of this route. A more precise description of the way the investigators recruited the participants would also result in a clearer description of the study population.

In our opinion, patients with a new episode of chronic LBP who report to a general practitioner might well differ from patients with a long history of LBP seen by a chiropractor. If all 4 methods of recruitment provided similar numbers of participants, we doubt whether the studied popu- lation is a representative sample of the LBP population presenting in general clinical practice.

Jos Runhaar, MSc

j.runhaar@erasmusmc.nl

Johannes C. van der Wouden, PhD

Department of General Practice

Erasmus MC-University Medical Center Rotterdam

Rotterdam, the Netherlands

Financial Disclosures: None reported.

1. Wilkens P, Scheel IB, Grundnes O, Hellum C, Storheim K. Effect of glucosamine on pain-related disability in patients with chronic low back pain and degenerative lumbar osteoarthritis: a randomized controlled trial. JAMA. 2010; 304(1):45-52.

In Reply: Drs Runhaar and van der Wouden raise interesting points about patient population generalizability in our clinical trial. In our view, patients included in our study were representative of patients seen in general clinical practice. Recruitment was $16.4 \%$ by general practitioners, $52.0 \%$ from chiropractors, $12.0 \%$ from physiotherapists, and $19.6 \%$ from the newspaper advertisement. In Norway, persons with chronic LBP with or without an acute flare often see different types of health care personnel according to their own preferences. Medical doctors, chiropractors, and physiotherapists specializing in manual therapy are licensed to prescribe sick leave; order x-ray tests, computed tomography, and MRIs; and refer to medical specialists.

Therefore, the difference between patients attending general practitioners and patients attending chiropractors or physiotherapists is probably limited. To our knowledge, there is no evidence supporting a notion of patient difference based on health care personnel. Thus, in terms of recruitment, we consider our group of selected patients generalizable to general clinical practice.

We also believe that recruitment from different health care personnel strengthens the generalizability of the population, which is principally a function of the inclusion and exclusion criteria used to include the appropriate patients. Additionally, regardless of path into the study, the majority of the patients had visited their primary physicians and 1 or more physiotherapists or chiropractors recently or in the past.

All patients were required to have had an MRI, not only the self-referred patients. In Norway, the public health insurance agency assumes the cost of the MRI, apart from a minor out-of-pocket fee of approximately $\$ 40$.

Philip Wilkens, MChiro

philip.wilkens@medisin.uio.no

Department of Orthopedics

Oslo University Hospital

Oslo, Norway

Inger B. Scheel, PhD

SINTEF Health Research

Oslo

Kjersti Storheim, PhD

Department of Orthopedics

Oslo University Hospital

Financial Disclosures: None reported. 